\title{
Spatiotemporal expansion of human brucellosis in Shaanxi Province, Northwestern China and model for risk prediction
}

\author{
Zurong Yang Equal first author, 1, 2 , Miaomiao Pang Equal first author, 3 , Qingyang Zhou ${ }^{2}$, Shuxuan Song ${ }^{1}$, Weifeng Liang ${ }^{4}$, Junjiang \\ Chen ${ }^{1}$, Tianci Guo ${ }^{1}$, Zhongjun Shao ${ }^{\text {Corresp., } 1}$, Kun Liu ${ }^{\text {Corresp. } 1}$ \\ 1 Department of Epidemiology, Ministry of Education Key Lab of Hazard Assessment and Control in Special Operational Environment, School of Public \\ Health, Air Force Medical University, Xi'an, Shaanxi, People's Republic of China \\ 2 Centre for Disease Prevention and Control in Northern Theater Command, Shenyang, People's Republic of China \\ ${ }^{3}$ Shaanxi Provincial Corps Hospital of Chinese People's Armed Police Force, Xi'an, Shaanxi, People's Republic of China \\ 4 Health Commission of Shaanxi Province, Xi'an, Shaanxi, People's Republic of China \\ Corresponding Authors: Zhongjun Shao, Kun Liu \\ Email address: 13759981783@163.com, liukun5959@fmmu.edu.cn
}

Background. Human brucellosis imposes a heavy burden on the health and economy of endemic regions. Since 2011, China has reported at least 35,000 human brucellosis cases annually, with more than $90 \%$ of these cases reported in the northern. Given the alarmingly high incidence and variation in the geographical distribution of human brucellosis cases, there is an urgent need to decipher the causes of such variation in geographical distribution. Method. We conducted a retrospective epidemiological study in Shaanxi Province from January 1, 2005 to December 31, 2018 to investigate the association between meteorological factors and transmission of human brucellosis according to differences in geographical distribution and seasonal fluctuation in northwestern China for the first time. Results. Human brucellosis cases were mainly distributed in the Shaanbei upland plateau before 2008 and then slowly extended towards the southern region with significant seasonal fluctuation. The results of quasi-Poisson generalized additive mixed model (GAMM) indicated that air temperature, sunshine duration, rainfall, relative humidity, and evaporation with maximum lag time within 7 months played crucial roles in the transmission of human brucellosis with seasonal fluctuation. Compared with the Shaanbei upland plateau, Guanzhong basin had more obvious fluctuations in the occurrence of human brucellosis due to changes in meteorological factors. Additionally, the established GAMM model showed high accuracy in predicting the occurrence of human brucellosis based on the meteorological factors.

Conclusion. These findings may be used to predict the seasonal fluctuations of human brucellosis and to develop reliable and cost-effective prevention strategies in Shaanxi Province and other areas with similar environmental conditions. 


\section{Spatiotemporal expansion of human brucellosis in}

2 Shaanxi Province, Northwestern China and model for

3 risk prediction

4

Zurong Yang ${ }^{1,2 \#}$, Miaomiao Pang ${ }^{3 \#}$, Qingyang Zhou ${ }^{2}$, Shuxuan Song ${ }^{1}$, Weifeng Liang ${ }^{4}$, Junjiang Chen ${ }^{1}$, Tianci Guo ${ }^{1}$, Zhongjun Shao ${ }^{1}$, Kun Liu ${ }^{1}$

${ }^{1}$ Department of Epidemiology, Ministry of Education Key Lab of Hazard Assessment and Control in Special Operational Environment, School of Public Health, Air Force Medical University, Xi'an, Shaanxi, People's Republic of China

${ }^{2}$ Centre for Disease Prevention and Control in Northern Theater Command, Shenyang, Liaoning, People's Republic of China

${ }^{3}$ Shaanxi Provincial Corps Hospital of Chinese People's Armed Police Force, Xi'an, Shaanxi, People's Republic of China

${ }^{4}$ Health Commission of Shaanxi Province, Xi'an, Shaanxi, People's Republic of China

\# These authors contributed equally to this work

Corresponding Author:

Zhongjun Shao, Kun Liu

169 West Changle Road, Xi'an, Shaanxi, 700032, People's Republic of China

Email address: 13759981783@163.com, liukun5959@qq.com

\section{Abstract}

Background. Human brucellosis imposes a heavy burden on the health and economy of endemic regions. Since 2011, China has reported at least 35,000 human brucellosis cases annually, with more than $90 \%$ of these cases reported in the northern. Given the alarmingly high incidence and variation in the geographical distribution of human brucellosis cases, there is an urgent need to decipher the causes of such variation in geographical distribution.

Method. We conducted a retrospective epidemiological study in Shaanxi Province from January 1, 2005 to December 31, 2018 to investigate the association between meteorological factors and transmission of human brucellosis according to differences in geographical distribution and seasonal fluctuation in northwestern China for the first time.

Results. Human brucellosis cases were mainly distributed in the Shaanbei upland plateau before 2008 and then slowly extended towards the southern region with significant seasonal fluctuation. The results of quasi-Poisson generalized additive mixed model (GAMM) indicated that air temperature, sunshine duration, rainfall, relative humidity, and evaporation with maximum lag time within 7 months played crucial roles in the transmission of human brucellosis with seasonal fluctuation. Compared with the Shaanbei upland plateau, Guanzhong basin had more obvious fluctuations in the occurrence of human brucellosis due to changes in meteorological factors. 
40 Additionally, the established GAMM model showed high accuracy in predicting the occurrence 41 of human brucellosis based on the meteorological factors.

42 Conclusion. These findings may be used to predict the seasonal fluctuations of human

43 brucellosis and to develop reliable and cost-effective prevention strategies in Shaanxi Province 44 and other areas with similar environmental conditions. 


\section{Introduction}

46 Brucellosis is a bacterial zoonosis caused by genus Brucella, including B. abortus, B. canis, B. individual-to-individual transmission, the environment-to-individual transmission is more common (Li et al., 2017), which means that most people are infected by contacting with Brucella in the environment, such as contaminated forage, water, grass, liquids, products, raw milk and the uterine fluids from infected animals (Li et al., 2017; Nematollahi et al., 2017; Chen et al., 2013; Liu et al., 2020). Thus, shepherds, breeders, abattoirs workers, veterinarians and laboratory personnel who potentially contact the bacteria are at high risks (Chen et al., 2016). Infected people present a variety of symptoms such as night sweats, arthralgia, undulant fever, hepatomegaly, headaches, myalgia, and personality changes (Wang et al., 2020; Lou et al., 2016; Li et al., 2013). Since most infected people go to the clinic for treatment only when they have clinical symptoms such as undulant fever, asymptomatic infections often missed and/or misdiagnosed (Zhen et al., 2013). In addition, low awareness, ineffective preventative measures, high initial treatment failure, substantial residual disability, and relapse rates have contributed to the heavy burden of this disease on the health and economy of endemic regions (Lai et al., 2017). Globally, the World Health Organization (WHO) and the Food and Agriculture Organization of the United Nations (FAO) announced that brucellosis is one of the most significantly neglected zoonotic diseases in the world (Dadar et al., 2019). The virus results in tremendous economic losses and health threats in countries whose economies are dominated by livestock keeping. Human brucellosis is epidemic in many countries especially in Latin America, Middle East, and South and Central Asia, with a total of cases more than 500,000 cases each year (Zhao et al., 2019). Since 2011, 31 provinces in China have reported at least 35,000 human brucellosis cases annually, with more than $90 \%$ of these cases reported in northern China (Wang et al., 2013). Nationwide, the incidence of human brucellosis shows an apparent geographic expansion from northern pastureland provinces to the adjacent grassland and agricultural areas, then to southern coastal and southwestern areas (Guan et al., 2018; Yang et al., 2020). From 2005 to 2018, a total of 12,671 confirmed human brucellosis cases were reported in Shaanxi Province, with approximately $70.36 \%$ of cases reported in Shaanbei upland plateau, and the average annual incidence reached 11.50/100,000, which was higher than the national average incidence in 2018 $(2.73 / 100,000)$ (Pang et al., 2020). Given the alarmingly high incidence and variation in the geographical distribution of human brucellosis cases, there is an urgent need to decipher the causes of such variation in geographical distribution.

The aim of the current study was to examine the spatial and temporal distributions of human brucellosis in Shaanxi Province between 2005 and 2018. We also explored the associations between meteorological factors and the environment-to-individual transmission of human brucellosis using a quasi-Poisson generalized additive mixed model (GAMM), and then analyzed the driving effect of meteorological factors on the distribution of human brucellosis and predicted the short-term incidence trend in the main epidemic areas of Shaanxi, northwestern 4 China. 
85

86

87

88

89

90

91

92

93

94

95

96

97

98

99

100

101

102

103

104

105

106

107

108

109

110

111

112

113

114

115

116

117

118

119

120

121

122

123

\section{Materials \& Methods}

\section{Study Region}

Shaanxi is a northwestern administrative province of China, extending from $31^{\circ} 42^{\prime}$ $39^{\circ} 35^{\prime} \mathrm{N}$ to $105^{\circ} 29^{\prime}-115^{\circ} 15^{\prime}$ E. It consists of 11 prefecture-level cities including 30 municipal districts and 77 counties with an area of $205,800 \mathrm{~km}^{2}$ and a total population of 3.86 million in 2018 (http://tjj.shaanxi.gov.cn/index.htm). According to the typical climate and landforms, Shaanxi Province can be divided into three distinct natural sub-regions: the Shaanbei upland plateau (northern Shaanxi), the Guanzhong basin (middle part of Shaanxi), and the Shaannan mountainous region (southern Shaanxi) (Figure S1). The Shaanbei upland plateau includes two cities (Yulin and Yan'an) and is part of the transitional landscape spanning from the Maowusu Desert to the Loess Plateau, which belongs to a typical crisscross zone of livestock keeping and farming. This region has constantly been one of the most severely affected areas by brucellosis in China (Chen et al., 2013), and the nearby provinces (Inner Mongolia, Gansu, Ningxia, and Shanxi) have all reported a high prevalence of brucellosis (Lai et al., 2017; Wang et al., 2013). The Guanzhong basin is located in the middle of Shaanxi province and mainly comprises plains and river landforms. This region has a large population with a huge demand for milk and meat products; therefore, transportation and livestock trade are active in this region. In recent years, the epidemic of brucellosis showed a rapid increasing trend and mainly affected people with occupational exposures, such as farmers, market workers, slaughterhouse workers, and veterinarians (Chen et al., 2016; Zhao et al., 2019). The Shaannan mountainous region is characterized by mountains and hills with a subtropical and continental monsoon climate. Sporadic human brucellosis cases have been reported in the region.

\section{Data Collection}

In China, human brucellosis is listed as a class B notifiable infectious disease, and reporting of data for diagnosed cases (including age, gender, occupation, address and date of brucellosis onset) to the local Center for Disease Control and Prevention (CDC) through the National Notifiable Infectious Diseases Reporting Information System is mandatory. For this study, we obtained the data of human brucellosis cases in Shaanxi from January 1, 2005 to December 31, 2018 from the CDC of Shaanxi Province. All cases were diagnosed based on a combination of epidemiologic exposures, clinical features (fever lasting several days or weeks, sweating, fatigue, and muscle or joint pain, e.g.), and serological test results. Confirmatory tests including the standard plate agglutination test (PAT), rose bengal plate test, serum agglutination test, or isolation of Brucella spp were further conducted to confirm the human brucellosis cases. In addition, the demographic data from the 6th census conducted by the National Bureau of Statistics of China in 2010 were used to calculate the incidence of human brucellosis. The local monthly meteorological data for air temperature $\left({ }^{\circ} \mathrm{C}\right)$, evaporation $(\mathrm{mm})$, rainfall $(\mathrm{mm})$, sunshine duration (h) and relative humidity (\%) for the study period were obtained from the Chinese Bureau of Meteorology (http://data.cma.cn/). 


\section{Statistical Analysis}

125 We determined the spatiotemporal distributions of human brucellosis in Shaanxi Province 126 between 2005 and 2018 with stratification for three sub-regions, and the monthly accumulative 127 incidence of brucellosis was geo-referenced to each county on a digital map. In addition, we used 128 a time-series analysis method to identify the relationships and potential effects between 129 meteorological factors and the incidence of human brucellosis. Specifically, we utilized the 130 cross-correlation analysis to assess the associations between meteorological factors and human 131 brucellosis for a range of lags up to 7 months and presented meteorological factors with the 132 maximum correlation coefficients in areas with high incidences (Shaanbei upland plateau and 133 Guanzhong basin). Finally, to identify potential non-linear relationships between meteorological 134 factors and the monthly incidence of human brucellosis, we applied cubic spline analysis 135 including those variables from the quasi-Poisson GAMM model; the model was performed to 136 examine the independent contribution of meteorological factors to the transmission of human 137 brucellosis (Cao et al., 2020; Sun et al., 2018). To adjust for long-term trends, seasonality, and 138 over-dispersion, we selected an appropriate degree of freedom (df) and lag for each variable in 139 the model (Duan et al., 2017; Zhang et al., 2010). The structure of the model used in the current 140 study is shown below:

$$
\log \left(\mathrm{Y}_{\mathrm{t}}\right)=\beta_{0}+\text { Group }_{+} s(\text { incidence,month })+\sum s\left(\text { variables }_{t-\mathrm{e}}, \text { by }=\text { Group, } \mathrm{df}\right),
$$

142

143

144

145

146

147

148

149

150

151

152

153

154

155

156

157

158

159

160

161

162

163

where $Y_{t}$ is the number of human brucellosis cases in month $\mathrm{t} ; \beta_{0}$ is the intercept; Group is used to control regional difference; $\mathrm{s}$ (incidence,month) exploits the link between incidence and month to control random effects; $\sum \mathrm{s}$ (variables $_{t-\mathrm{e}^{\prime}}$ by $=$ Group,df $)$ denotes the cubic spline of meteorological factors, including air temperature, evaporation, rainfall, sunshine duration and relative humidity, in the previous e months with corresponding $\mathrm{df}=12$ for different region. The $\mathrm{R}$ squared value, deviance explained value, and generalized cross-validation (GCV) estimation value were used to determine the most appropriate model.

The statistical analyses were performed using R software version 3.6.0 with the packages of "mgcv" and "itsadug". The spatial analyses were performed using ArcGIS 10.2 Software (ESRI Inc.; Redlands, CA, USA). All statistical tests were two-sided, and a $p$ value $<0.05$ was considered statistically significant.

\section{Results}

\section{Spatial and Temporal Distributions of Human Brucellosis}

Between 2005 and 2018, a total of 12,671 confirmed human brucellosis cases were reported in Shaanxi Province with significant geographical heterogeneity. Nearly $70.36 \%$ of these cases $(8,916)$ were reported in Shaanbei upland plateau, and approximately $28.06 \%$ cases $(3,555)$ were reported in Guanzhong basin. Only 200 cases (1.58\%) were reported in Shaannan mountainous region. The annual number of cases of human brucellosis in Shaanxi fluctuated from 585 (1.57 per 100,000 persons) in 2005 to 673 (1.80 per 100,000 persons) in 2018, with two peaks in 2008 (1,238 cases, 3.32 per 100,000 persons) and 2014 (1,542 cases, 4.13 per 100,000 persons) (Figure 1). When stratified by the three sub-regions, the annual number of cases in Shaanbei 
164 upland plateau showed bimodal peaks with a major peak in 2008 (1,104 cases, 19.93 per 100,000

165

166

167

168

169

170

171

172

173

174

175

176

177

178

179

180

181

182

183

184

185

186

187

188

189

190

191

192

193

194

195

196

197

198

199

200

201

202

203

persons) and a minor peak in 2014 (794 cases, 14.34 per 100,000 persons); however, the annual number of cases of Guanzhong basin gradually increased from 2008, peaking in 2014 with 729 cases (3.12 per 100,000 persons). The annual number of cases of Shaannan mountainous region slowly increased as well, peaking in 2015 with 83 cases ( 0.99 per 100,000 persons) (Figure 2). The spatial distribution of human brucellosis showed that most cases occurred in Shaanbei upland plateau before 2008, with gradual expansion to middle and southern region (Figure 3A). Notably, Dali county and Chengcheng county, which were located in low incidence areas, were sites of outbreaks in 2014. The monthly accumulative incidence showed seasonal fluctuations (Figure 3B), with $60.36 \%(7,648)$ of cases occurring between March and July. Sporadic human brucellosis cases occurred in numerous regions of Shaannan mountainous region without significant seasonal fluctuations. Further analysis found that the peak of human brucellosis in Shaanbei upland plateau occurred in March to July, but Guanzhong basin occurred in April to July (Figure 3C and 3D). In addition, Zizhou County reported the highest annual incidence (153.46 per 100,000 persons) in 2008 and the highest monthly accumulative incidence (104.03 per 100,000 persons) in April.

\section{Correlations between Human Brucellosis Incidence and Meteorological Factors}

Shaanbei upland plateau and the Guanzhong basin had the highest numbers of human brucellosis cases in Shaanxi as major endemic area. Figure S 2A\&2B showed the monthly incidence of human brucellosis in Shaanbei upland plateau was periodic and seasonal fluctuations. In contrast, the periodic and seasonal fluctuations of monthly incidence in Guanzhong basin gradually became obvious, as the incidence increased. The mean air temperature, sunshine duration, evaporation illustrated showed a similar fluctuation with the monthly incidence, while for rainfall and relative humidity an opposite trend is observed. Therefore, correlations between meteorological factors and the number of human brucellosis cases were further explored in these two regions (Table 1). The maximum correlation coefficients for meteorological factors, including air temperature, rainfall, relative humidity, sunshine duration and evaporation, showed lag time of 4 months, 3 months, 2 months, 6 months, and 5 months in Shaanbei upland plateau, respectively. However, the maximum correlation coefficients for the abovementioned meteorological factors showed lag time of 5 months, 4 months, 3 months, 7 months, and 6 months in the Guanzhong basin, respectively, which all lagged 1 month behind those in Shaanbei upland plateau. According to our correlation analysis between meteorological factors and monthly number of reported cases in the two regions, air temperature had a relatively stronger correlation than other factors, with Spearman correlation coefficients of 0.56 and 0.36 in Shaanbei upland plateau and the Guanzhong basin, respectively (Table 1 and Figure S 3A\&3B). The mean values for the monthly number of human brucellosis cases and meteorological factors in Shaanbei upland plateau and the Guanzhong basin are presented in Table 2. From 2005 to 2018 in Shaanbei upland plateau, the mean values for monthly cases, air temperature, relative humidity, cumulative rainfall, cumulative evaporation, and cumulative sunshine duration were 53.07 cases, $9.90^{\circ} \mathrm{C}, 55.53 \%, 40.78 \mathrm{~mm}, 122.86 \mathrm{~mm}$, and $215.87 \mathrm{~h}$, respectively. The

Peer) reviewing PDF | (2020:05:49509:1:1:NEW 15 Aug 2020) 
204 corresponding mean values in the Guanzhong basin were 21.16 cases, $12.10^{\circ} \mathrm{C}, 65.16 \%, 50.79$

$205 \mathrm{~mm}, 101.97 \mathrm{~mm}$, and $171.90 \mathrm{~h}$, respectively.

206 Estimation of Meteorological Effects on Human Brucellosis in Shaanbei Upland Plateau and 207 Guanzhong Basin

208 To analyze the seasonal fluctuation of human brucellosis of Shaanbei upland plateau and the 209 Guanzhong basin, a quasi-Poisson GAMM model was used. After controlling for auto210 correlation, seasonality, and the lag effect, we found that the monthly number of human 211 brucellosis cases was significantly associated with previous cases and meteorological factors 212 (most $\mathrm{p}$ values $<0.05$ ), including air temperature, relative humidity, rainfall, evaporation, and 213 sunshine duration (Table 3). According to the values for R square (0.90), deviance explained 214 (91.00\%), and GCV principles (4.32), the most appropriate GAMM model was fitted and 215 selected. The observed and fitted cases from the final model matched relatively well for 216 Shaanbei upland plateau and the Guanzhong basin, and the respective goodness of fit (R square) 217 values were $91.43 \%$ and $87.83 \%$, respectively. A good fit between observed cases and predicted 218 cases was achieved, using the 24-month observations, and the goodness-of-fit analyses showed 219 that the residuals did not cause significant auto-correlation in the final model (Figures 4A\&4B 220 and 5A\&5B). In addition, we found the effects of meteorological factors on the occurrence of 221 human brucellosis were different in Shaanbei upland plateau and Guanzhong basin through 222 Figure 6A-6E and Table 3. Compared with the Shaanbei upland plateau, Guanzhong basin had 223 more obvious fluctuations in the occurrence of human brucellosis due to changes in 224 meteorological factors, such as air temperature, relative humidity, rainfall, sunshine duration, and evaporation. The summed effects of the meteorological factors parameters terms in Guanzhong basin was 3.48 (95\% CI: 2.84-4.12), while the Shaanbei upland plateau was 2.94 (95\% CI: 2.703.20). The effect of the meteorological factors indicated that except for the air temperature of the current month and rainfall of the previous month showed an upward trend, and previous sunshine duration was U-shaped, the rest of the meteorological factors was a downward trend in Guanzhong basin. High air temperature, low relative humidity, less rainfall, short sunshine duration, and suitable evaporation in this region all had positive effects on the incidence of human brucellosis. In addition, such positive effects were observed for low air temperature and long sunshine duration of the previous month. However, only a weak trend can be observed in Shaanbei upland plateau. Furthermore, the identified interactions between two meteorological factors in association with human brucellosis are shown in Figure S4 A-J. The result showed the interaction between high air temperature and lower relative humidity, higher air temperature and shorter sunshine duration, lower relative humidity and shorter sunshine duration, lower relative humidity and suitable evaporation, and less rainfall and suitable evaporation were all obviously associated with high incidence of human brucellosis. Especially in the environment of high air temperature, low relative humidity and short sunshine duration, the risk of human brucellosis is higher. 


\section{Discussion}

244 To our best knowledge, this is the first comprehensive study to analyze the association between

245

246

247

248

249

250

251

252

253

254

255

256

257

258

259

260

261

262

263

264

265

266

267

268

269

270

271

272

273

274

275

276

277

278

279

280

281

282 meteorological factors and transmission of human brucellosis according to differences in geographical distribution and seasonal fluctuation. We found that human brucellosis cases were distributed mainly in the northern part of Shaanxi province before 2008 and then slowly extended towards south with obvious seasonal fluctuations. The quasi-Poisson GAMM model suggested that air temperature, sunshine duration, rainfall, relative humidity, and evaporation with lag time within 7 months may play a crucial role in the transmission, especially environment-to-individual transmission, of human brucellosis and the variation in geographical distribution, and the model had great accuracy in predicting the occurrence of human brucellosis. The results suggested that the established GAMM can accurately forecast the short-term incidence over 24 months based on meteorological factors, which has important public health implications for developing reliable and cost-effective prevention strategies, including vaccination time, reservoir surveillance, environment disinfection frequency, elimination rates of infected animals and medical resource allocation.

The higher incidences of human brucellosis in Shaanbei upland plateau corroborated with the observation that the northern part of China has historically experienced severe endemic area of brucellosis (Wang et al., 2013). In fact, the cases of human brucellosis reported in the northern part of China account for more than $90 \%$ of the total reported cases every year (Chen et al., 2013; Lai et al., 2017; Wang et al., 2013; Shi et al., 2017). Our study utilized a spatial-temporal overview and analysis of potential interactions between meteorological factors and found that the number of reported cases in Shaanbei upland plateau decreased gradually over the study period, while the number of reported cases in the Guanzhong basin increased rapidly. In addition, we observed that the peak of incidence and lag period of meteorological factors was 1 month earlier in Shaanbei upland plateau than Guanzhong basin. Therefore, we assumed that the Guanzhong basin has become a new endemic region of brucellosis, and imported cases have led to the occurrence of hysteresis.

In addition to the geographic difference, we observed seasonal fluctuation in the human brucellosis in the study (Figure 2 and Figure S2). Consistent with the previous finding that meteorological factors play a crucial role in the seasonal fluctuation and transmission of human brucellosis between reservoir and susceptible populations (Li et al., 2013; Zhao et al., 2019; Cao et al., 2020), our study found that air temperature, sunshine duration, relative humidity, rainfall, and evaporation were associated with the seasonal fluctuation and geographic variation in the incidence of human brucellosis. The metrological factors may influence the dynamics of reservoirs and viral transmission within a susceptible population; other factors such as types of land use, vegetation, and patterns of agricultural production are also possible contributors accounting for the observed association (Figure S3).

Short sunshine duration and low relative humidity may have contributed to the human brucellosis incidence through a few pathways. First, appropriate sunshine duration and relative humidity have significant effects on estrus of reservoirs, which is critical to the transmission

Peer] reviewing PDF | (2020:05:49509:1:1:NEW 15 Aug 2020) 
283 among susceptible herbs of brucellosis (Li et al., 2013; Cao et al., 2020). Second, most animals

284 infected with brucellosis do not show obvious signs, and transmission to the susceptible

285 population occurs mainly through abortion and secretion infection. With the arrival of the

286 production season, a large number of Brucella are excreted into the environment through the

287 abortive secretion of reservoirs. Meanwhile, a large number of susceptible lambs are bred and a

288 dry environment suitable for transmission of Brucella is established, which may lead to higher

289 risks of exposing to susceptible livestock and humans (Yang et al., 2018). In addition, low

290 rainfall and suitable evaporation have shown to exert negative impacts on vegetation growth that

291 serves as food for livestock (Cotterill et al., 2018). Therefore, our findings may suggest that

292 vegetation plays an important role in the transmission and variation of geographical distribution

293 of human brucellosis.

294 We hypothesized that several factors contributed to the observed trends in human brucellosis

295 cases. Shaanbei upland plateau has been a traditional endemic area for human brucellosis and has

296 experienced several outbreaks. Thus, public and governmental sectors may have strengthened

297 awareness of prevention and have adopted effective and targeted preventive measures. For

298 example, they may have promoted prevention and control of brucellosis in the public and

299 adopted strengthened quarantine practices in endemic areas. As a result, all these measures may

300 have led to the blockage of the transmission routes ( $\mathrm{Li}$ et al., 2017), the reduction in the

301 incidence, and the reduction of the impact of indirect factors such as meteorological factors.

302 However, the Guanzhong basin is a new endemic area for human brucellosis, and thus, local

303 sectors may have lacked experience and awareness in the prevention and control of the epidemic.

304 In addition, transportation and farming are active in this area, which may have influence on the

305 seasonal fluctuation. In the winter, the livestock and production activities closely related to

306 infected livestock such as cows and sheep are less active. In comparison, in the warm spring and

307 summer seasons, the activities related to farming and production (e.g.; lamb delivery, lamb

308 breeding, handling aborted placenta, shearing wool, and processing and trading meat products)

309 that are closely related to infected reservoirs may increase the exposure frequency of the local

310 population to the virus existing in infected reservoirs and contaminated products. Furthermore,

311 high air temperature has been shown to have a positive effect on the persistence and transmission

312 of Brucella (Lee et al., 2013), which is likely to infect susceptible herbs and cause epidemics

313 when protection and awareness are insufficient.

314 Some limitations of the current study merit consideration. Although the data quality from the

315 National Notifiable Disease Surveillance System is expected to be highly credible,

316 underreporting of cases due to mild or unnoticeable clinical symptoms may still exist. In

317 addition, the forecast model constructed in the current study was based on meteorological factors

318 only and used to predict short-term incidence. Third, we did not have data on other factors that

319 may influence the transmission of human brucellosis such as dietary habits, tourism, and

320 traveling (Zhu et al., 2017); thus, residual confounding factors may exist that could affect the

321 interpretation of the results. Finally, that is necessary to confirm part areas of Guanzhong basin 
322

323

324

325

326

327

328

329

330

331

332

333

334

335

336

337

338

339

340

341

342

343

344

345

346

347

348

349

350

351

352

353

354

355

356

357

358

359

360

361

362

363

364

whether has become endemic region of brucellosis through testing of susceptible animals in key areas.

\section{Conclusions}

The current study implies that meteorological factors may make important contributions to the transmission and variation in geographical distribution of human brucellosis by affecting the external environment, susceptible livestock and/or human populations, activity of reservoirs or the susceptible population, and vegetation. The established GAMM forecast model was shown to be accurate and applicable for predicting the seasonal fluctuation of human brucellosis. Further studies are warranted to validate our findings and develop reliable and cost-effective prevention strategies to combat human brucellosis.

\section{Acknowledgements}

We are grateful to every medical staff struggling on the brucellosis prevention and control, especially those who have contributed to the reporting of human brucellosis in Shaanxi Province.

\section{References}

Dadar M, Shahali Y, and Whatmore AM. 2019. Human brucellosis caused by raw dairy products: A review on the occurrence, major risk factors and prevention. International Journal of Food Microbiology 292:39-47. 10.1016/j.ijfoodmicro.2018.12.009.

Li MT, Sun GQ, Zhang WY, Jin Z. 2017. Model-Based Evaluation of Strategies to Control Brucellosis in China. International Journal of Environmental Research and Public Health 14. 10.3390/ijerph14030295.

Nematollahi S, Ayubi E, Karami M, Khazaei S, Shojaeian M, Zamani R, Mansori K, and Gholamaliee B. 2017. Epidemiological characteristics of human brucellosis in Hamadan Province during 2009-2015: results from the National Notifiable Diseases Surveillance System. International Journal of Infectious Diseases 61:56-61. 10.1016/j.ijid.2017.06.002.

Chen Z, Zhang W, Ke Y, Wang Y, Tian B, Wang D, Cui B, Zou W, Li S, Huang L, and Song H. 2013. High-risk regions of human brucellosis in china: implications for prevention and early diagnosis of travel-related infections. Clinical Infectious Diseases 57:330-332. $10.1093 / \mathrm{cid} / \mathrm{cit} 251$.

Liu ZG, Wang M, Ta N, Fang MG, Mi JC, Yu RP, Luo Y, Cao X, and Li ZJ. 2020. BrucellaSeroprevalence of human brucellosis and molecular characteristics of strains in Inner Mongolia Autonomous region of China, from 2012 to 2016. Emerging microbes \& infections 9:263-274. 10.1080/22221751.2020.1720528.

Chen Q, Lai S, Yin W, Zhou H, Li Y, Mu D, Li Z, Yu H, and Yang W. 2016. Epidemic characteristics, high-risk townships and space-time clusters of human brucellosis in Shanxi Province of China, 2005-2014. BMC Infectious Diseases 16:760. 10.1186/s12879-0162086-x.

Wang H, Hoffman C, Yang X, Clapp B, and Pascual DW. 2020. Targeting resident memory T cell immunity culminates in pulmonary and systemic protection against Brucella infection. PLoS Pathogens 16:e1008176. 10.1371/journal.ppat.1008176.

Lou P, Wang L, Zhang X, Xu J, and Wang K. 2016. Modelling Seasonal Brucellosis Epidemics 
365

366

367

368

369

370

371

372

373

374

375

376

377

378

379

380

381

382

383

384

385

386

387

388

389

390

391

392

393

394

395

396

397

398

399

400

401

402

403

404

405

406

407

408

409

410

in Bayingolin Mongol Autonomous Prefecture of Xinjiang, China, 2010-2014. BioMed res int 2016:5103718. 10.1155/2016/5103718.

Li YJ, Li XL, Liang S, Fang LQ, and Cao WC. 2013. Epidemiological features and risk factors associated with the spatial and temporal distribution of human brucellosis in China. BMC Infectious Diseases 13:547. 10.1186/1471-2334-13-547.

Zhen Q, Lu Y, Yuan X, Qiu Y, Xu J, Li W, Ke Y, Yu Y, Huang L, Wang Y, and Chen Z. 2013. Asymptomatic brucellosis infection in humans: implications for diagnosis and prevention. Clin microb inf 19:E395-397. 10.1111/1469-0691.12250.

Lai S, Zhou H, Xiong W, Gilbert M, Huang Z, Yu J, Yin W, Wang L, Chen Q, Li Y, Mu D, Zeng L, Ren X, Geng M, Zhang Z, Cui B, Li T, Wang D, Li Z, Wardrop NA, Tatem AJ, and Yu H. 2017. Changing Epidemiology of Human Brucellosis, China, 1955-2014. Emerging Infectious Diseases 23:184-194. 10.3201/eid2302.151710.

Zhao Y, Li R, Qiu J, Sun X, Gao L, and Wu M. 2019. Prediction of Human Brucellosis in China Based on Temperature and NDVI. International Journal of Environmental Research and Public Health 16. 10.3390/ijerph16214289.

Wang Y, Zhang W, Ke Y, Zhen Q, Yuan X, Zou W, Li S, Sun Y, Wang Z, Wang D, Cui B, Song H, Huang L, and Chen Z. 2013. Human brucellosis, a heterogeneously distributed, delayed, and misdiagnosed disease in china. Clinical Infectious Diseases 56:750-751. 10.1093/cid/cis980.

Guan P, Wu W, and Huang D. 2018. Trends of reported human brucellosis cases in mainland China from 2007 to 2017: an exponential smoothing time series analysis. Environmental Health and Preventive Medicine 23:23. 10.1186/s12199-018-0712-5.

Yang HX, Zhang SW, Wang TJ, Zhao CH, Zhang XY, Hu J, Han CY, Hu FF, Luo JJ, Li B, Zhao W, Li KW, Wang Y, and Zhen Q. 2020. Epidemiological Characteristics and Spatiotemporal Trend Analysis of Human Brucellosis in China, 1950-2018. International Journal of Environmental Research and Public Health 17. 10.3390/ijerph17072382.

Peng C, Zhou H, Guan P, Wu W, and Huang DS. 2020. An estimate of the incidence and quantitative risk assessment of human brucellosis in mainland China. Transboundary and Emerging Diseases. 10.1111/tbed.13518.

Cao LT, Liu HH, Li J, Yin XD, Duan Y, and Wang J. 2020. Relationship of meteorological factors and human brucellosis in Hebei province, China. Sci Total Environ 703:135491. 10.1016/j.scitotenv.2019.135491.

Sun JM, Lu L, Liu KK, Yang J, Wu HX, and Liu QY. 2018. Forecast of severe fever with thrombocytopenia syndrome incidence with meteorological factors. Sci Total Environ 626:1188-1192. 10.1016/j.scitotenv.2018.01.196.

Duan Y, Yang LJ, Zhang YJ, Huang XL, Pan GX, and Wang J. 2017. Effects of meteorological factors on incidence of scarlet fever during different periods in different districts of China. Sci Total Environ:19-24. 10.1016/j.scitotenv.2017.01.010.

Zhang WY, Guo WD, Fang LQ, Li CP, Bi P, Glass GE, Jiang JF, Sun SH, Qian Q, Liu W, Yan L, Yang H, Tong SL, and Cao WC. 2010. Climate variability and hemorrhagic fever with renal syndrome transmission in Northeastern China. Environmental Health Perspectives 118:915-920. 10.1289/ehp.0901504.

Shi YJ, Lai SJ, Chen QL, Mu D, Li Y, Li XX, Yin WW, and Yu HJ. 2017. Analysis on the epidemiological features of human brucellosis in northern and southern areas of China, 2015-2016. Zhonghua Liu Xing Bing Xue Za Zhi 38:435-440. 10.3760/cma.j.issn.02546450.2017.04.005. 
Yang ZR, Li X, Shao ZJ, Ma WT, Yuan XJ, Wu KJ, and Liu K. 2018. Characteristics on spatial and temporal distribution as well as the driving effect of meteorological factors on brucellosis in Datong city, Shanxi province, 2005-2015. Zhonghua Liu Xing Bing Xue Za Zhi 39:1165-1171. 10.3760/cma.j.issn.0254-6450.2018.09.005.

Cotterill GG, Cross PC, Cole EK, Fuda RK, Rogerson JD, Scurlock BM, and Biological sciences du Toit JT \%J Philosophical transactions of the Royal Society of London. Series B. 2018. Winter feeding of elk in the Greater Yellowstone Ecosystem and its effects on disease dynamics. Philos Trans R Soc Lond B, Biol Sci 373. 10.1098/rstb.2017.0093.

Lee HS, Her M, Levine M, and Moore GE. 2013. Time series analysis of human and bovine brucellosis in South Korea from 2005 to 2010. Preventive Veterinary Medicine 110:190197. 10.1016/j.prevetmed.2012.12.003.

Zhu HS, Wang LL, Lin DH, Hong RT, Ou JM, Chen W, Wu BP, Huang WL, Xie ZH, Chen GM, Wu SG, Xu ZB, and Deng YQ. 2017. Analysis on epidemiology and spatialtemporal clustering of human brucellosis in Fujian province, 2011-2016. Zhonghua Liu Xing Bing Xue Za Zhi 38:1212-1217. 10.3760/cma.j.issn.0254-6450.2017.09.014. 


\section{Table $\mathbf{1}$ (on next page)}

Table 1. Correlation analysis between meteorological factors and the number of human brucellosis cases in Shaanbei upland plateau and the Guanzhong basin in Shaanxi Province, China, 2005-2018.

${ }^{*} p<0.05,{ }^{* *} p<0.001$. 
1 Table 1. Correlation analysis between meteorological factors and the number of human brucellosis cases in Shaanbei upland plateau 2 and the Guanzhong basin in Shaanxi Province, China, 2005-2018.

\begin{tabular}{|c|c|c|c|c|c|c|}
\hline \multirow[b]{2}{*}{ Meteorological factors } & \multicolumn{3}{|c|}{ Shaanbei upland plateau } & \multicolumn{2}{|c|}{ Guanzhong basin } & \multirow[b]{2}{*}{$\mathrm{p}$} \\
\hline & $\begin{array}{l}\text { Spearman } \\
\text { coefficient }\end{array}$ & $\begin{array}{l}\text { Lag value } \\
\text { (month) }\end{array}$ & $\mathrm{p}$ & $\begin{array}{l}\text { Spearman } \\
\text { coefficient }\end{array}$ & $\begin{array}{l}\text { Lag value } \\
\text { (month) }\end{array}$ & \\
\hline Air temperature & $0.56^{* *}$ & 0 & $<0.001$ & $0.36^{* *}$ & 0 & $<0.001^{* *}$ \\
\hline Rainfall & $0.38^{* *}$ & 0 & $<0.001$ & $0.25^{* *}$ & 0 & $0.010^{*}$ \\
\hline Evaporation & $0.67^{* *}$ & 0 & $<0.001$ & $0.30^{* *}$ & 0 & $<0.001^{* *}$ \\
\hline Sunshine duration & $0.54^{* *}$ & 0 & $<0.001$ & $0.20^{*}$ & 0 & $0.010^{*}$ \\
\hline Humidity & $-0.22^{* *}$ & 0 & 0.01 & -0.06 & 0 & 0.460 \\
\hline Lag in air temperature & $-0.75^{* *}$ & 4 & $<0.001$ & $-0.40^{* *}$ & 5 & $<0.001^{* *}$ \\
\hline Lag in rainfall & $-0.59^{* *}$ & 3 & $<0.001$ & $-0.34^{* *}$ & 4 & $<0.001^{* *}$ \\
\hline Lag in evaporation & $-0.68^{* *}$ & 5 & $<0.001$ & $-0.37^{* *}$ & 6 & $<0.001^{* *}$ \\
\hline Lag in sunshine duration & $-0.50^{* *}$ & 6 & $<0.001$ & $-0.31^{* *}$ & 7 & $<0.001^{* *}$ \\
\hline Lag in humidity & $-0.66^{* *}$ & 2 & $<0.001$ & $-0.35^{* *}$ & 3 & $<0.001^{* *}$ \\
\hline
\end{tabular}




\section{Table 2 (on next page)}

Table 2. Summary of monthly numbers of human brucellosis cases and meteorological factors in Shaanbei upland plateau and the Guanzhong basin in Shaanxi Province, China, 2005-2018.

Min: minimum of the variable; P5: the 5th percentile of the variable; P25: the 25th percentile of the variable; P50: the 50th percentile of the variable; P75: the 75th percentile of the variable; P95: the 95th percentile of the variable; Max: maximum of the variable. SD: standard deviation. 
1 Table 2. Summary of monthly numbers of human brucellosis cases and meteorological factors in Shaanbei upland plateau and the

2 Guanzhong basin in Shaanxi Province, China, 2005-2018.

\begin{tabular}{|c|c|c|c|c|c|c|c|c|c|}
\hline \multicolumn{2}{|c|}{ Variables } & Min & P5 & $\mathrm{P} 25$ & P50 & P75 & P95 & Max & Mean \pm SD \\
\hline \multirow{6}{*}{ Shaanbei upland plateau } & Number of cases & 9.00 & 18.00 & 29.25 & 44.50 & 68.00 & 121.00 & 164.00 & $53.07 \pm 31.70$ \\
\hline & Mean air temperature $\left({ }^{\circ} \mathrm{C}\right)$ & -10.86 & -6.51 & 0.74 & 11.39 & 19.64 & 23.64 & 25.00 & $9.90 \pm 10.40$ \\
\hline & Mean relative humidity (\%) & 28.88 & 34.54 & 46.71 & 54.74 & 65.96 & 74.24 & 79.36 & $55.53 \pm 12.34$ \\
\hline & Rainfall (mm) & 0.00 & 0.35 & 6.89 & 22.30 & 60.35 & 124.67 & 301.60 & $40.78 \pm 45.41$ \\
\hline & Evaporation (mm) & 25.39 & 35.16 & 65.32 & 119.17 & 174.18 & 224.96 & 258.29 & $122.86 \pm 62.57$ \\
\hline & Sunshine duration $(\mathrm{h})$ & 106.44 & 139.14 & 190.46 & 219.22 & 244.82 & 279.98 & 292.00 & $215.87 \pm 40.87$ \\
\hline \multirow{6}{*}{ Guanzhong basin } & Number of cases & 0.00 & 1.00 & 7.00 & 15.00 & 30.75 & 57.55 & 111.00 & $21.16 \pm 19.92$ \\
\hline & Mean air temperature $\left({ }^{\circ} \mathrm{C}\right)$ & -5.51 & -2.07 & 3.92 & 13.23 & 21.08 & 24.64 & 26.55 & $12.10 \pm 19.92$ \\
\hline & Mean relative humidity (\%) & 39.37 & 46.77 & 57.43 & 64.48 & 73.08 & 82.15 & 86.34 & $65.16 \pm 10.51$ \\
\hline & Rainfall (mm) & 0.03 & 1.19 & 10.27 & 36.98 & 80.56 & 137.75 & 297.17 & $50.79 \pm 48.77$ \\
\hline & Evaporation (mm) & 27.33 & 36.72 & 57.32 & 94.73 & 140.94 & 189.66 & 269.21 & $101.97 \pm 49.46$ \\
\hline & Sunshine duration $(\mathrm{h})$ & 48.11 & 90.09 & 138.38 & 170.67 & 204.59 & 249.34 & 285.13 & $171.90 \pm 45.81$ \\
\hline
\end{tabular}

Min: minimum level of the variable; P5: 5th percentile of the variable; P25: 25th percentile of the variable; P50: 50th percentile of the variable; P75: 75th percentile of the variable; P95: 95th percentile of the variable; Max: maximum level of the variable; SD: standard deviation. 


\section{Table 3 (on next page)}

Table 3. Approximate significance of smooth terms in Shaanbei upland plateau and Guanzhong basin in Shaanxi Province, China, 2005-2018.

*Summary of the GAMM smooth terms, their p-values and effective degrees of freedom are listed. We constructed the final model based on current monthly meteorological factors, temperature lag of 4 months, rainfall lag of 3 months, relative humidity lag of 2 months, sunshine duration lag of 6 months and evaporation lag of 5 months in Shaanbei upland plateau. Meanwhile, we constructed the final model for the Guanzhong basin based on current monthly meteorological factors and previous meteorological factors with lag times 1 month greater than those in Shaanbei upland plateau. 
1 Table 3. Approximate significance of smooth terms in Shaanbei upland plateau and Guanzhong basin in Shaanxi Province, China, 2005-

22018.

\begin{tabular}{|c|c|c|c|c|}
\hline Variables & Regions & $\begin{array}{c}\text { Effective degrees of } \\
\text { freedom }\end{array}$ & $\mathrm{F}$ & $\mathrm{p}$ \\
\hline \multirow{2}{*}{$\mathrm{s}($ Air temperature $)$} & Guanzhong basin & 6.41 & 1.80 & 0.08 \\
\hline & Shaanbei upland plateau & 1.00 & 0.86 & 0.35 \\
\hline \multirow{2}{*}{$\mathrm{s}(\mathrm{Lag}$ in air temperature $)$} & Guanzhong basin & 7.73 & 1.49 & 0.15 \\
\hline & Shaanbei upland plateau & 1.59 & 4.34 & 0.01 \\
\hline \multirow{2}{*}{ s(Relative humidity) } & Guanzhong basin & 5.44 & 3.61 & $<0.001$ \\
\hline & Shaanbei upland plateau & 1.00 & 0.12 & 0.73 \\
\hline \multirow{2}{*}{$\mathrm{s}$ (Lag in relative humidity) } & Guanzhong basin & 6.62 & 4.06 & $<0.001$ \\
\hline & Shaanbei upland plateau & 1.00 & 4.21 & 0.04 \\
\hline \multirow{2}{*}{ s(Sunshine duration) } & Guanzhong basin & 2.37 & 2.71 & 0.04 \\
\hline & Shaanbei upland plateau & 1.75 & 3.18 & 0.04 \\
\hline \multirow{2}{*}{$\mathrm{s}$ (Lag in sunshine duration) } & Guanzhong basin & 9.41 & 1.66 & 0.09 \\
\hline & Shaanbei upland plateau & 1.00 & 4.64 & 0.03 \\
\hline \multirow{2}{*}{ s(Evaporation) } & Guanzhong basin & 3.47 & 4.60 & $<0.001$ \\
\hline & Shaanbei upland plateau & 2.31 & 1.63 & 0.21 \\
\hline \multirow{2}{*}{ s(Lag in evaporation) } & Guanzhong basin & 1.30 & 6.37 & 0.01 \\
\hline & Shaanbei upland plateau & 1.00 & 4.01 & 0.05 \\
\hline \multirow{2}{*}{$\mathrm{s}($ Rainfall $)$} & Guanzhong basin & 8.61 & 2.38 & 0.01 \\
\hline & Shaanbei upland plateau & 1.00 & 0.12 & 0.73 \\
\hline \multirow{2}{*}{$\mathrm{s}(\mathrm{Lag}$ in rainfall $)$} & Guanzhong basin & 8.23 & 1.75 & 0.08 \\
\hline & Shaanbei upland plateau & 1.00 & 0.42 & 0.52 \\
\hline
\end{tabular}

3 
Figure 1

Figure 1. Temporal distribution of human brucellosis in Shaanxi Province, China, 2005-2018.

The bar chart represents the monthly incidence of human brucellosis in Shaanxi, and the line represents the annual number of cases of human brucellosis in Shaanxi Province.

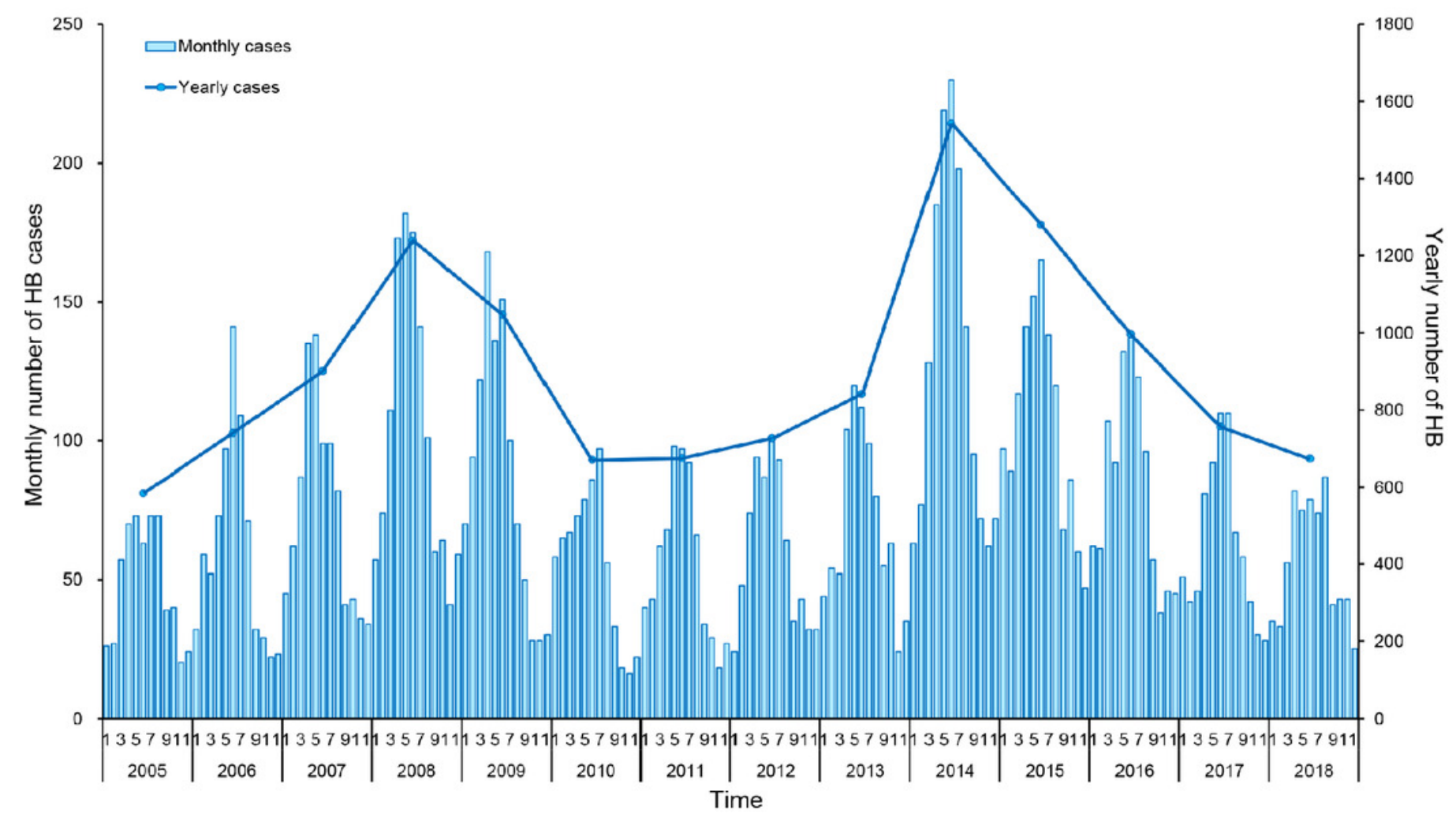


Figure 2

Figure 2. Temporal distribution of human brucellosis in Shaanbei upland plateau , Guanzhong basin, and Shaannan mountainous region, 2005-2018.

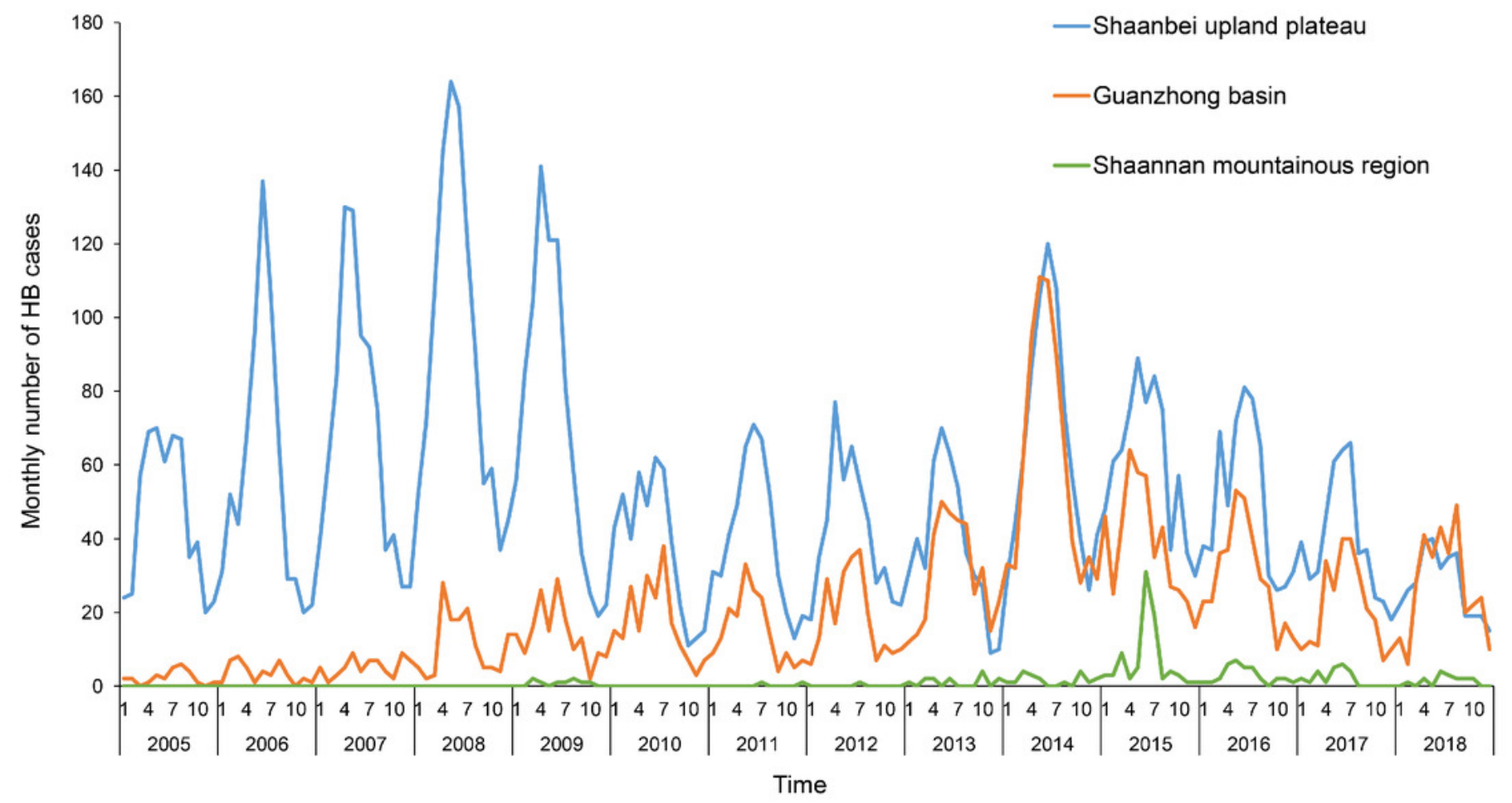


Figure 3

Figure 3. Spatial and temporal distribution of human brucellosis in Shaanxi Province.

(A) Annual incidence of human brucellosis in each county in Shaanxi Province, 2005-2018. The map was created by Zurong Yang in ArcGIS 10.1 Software, ESRI Inc., Redlands, CA, USA, (https://www.arcgis.com/index.html). (B) Spatial distribution of human brucellosis in Shaanxi Province from January to December, 2005-2018. The map was created by Zurong Yang in ArcGIS 10.1 Software, ESRI Inc., Redlands, CA, USA, (https://www.arcgis.com/index.html). (C) Seasonal distribution of human brucellosis in Shaanbei upland plateau. (D) Seasonal distribution of human brucellosis in Guanzhong basin.

A
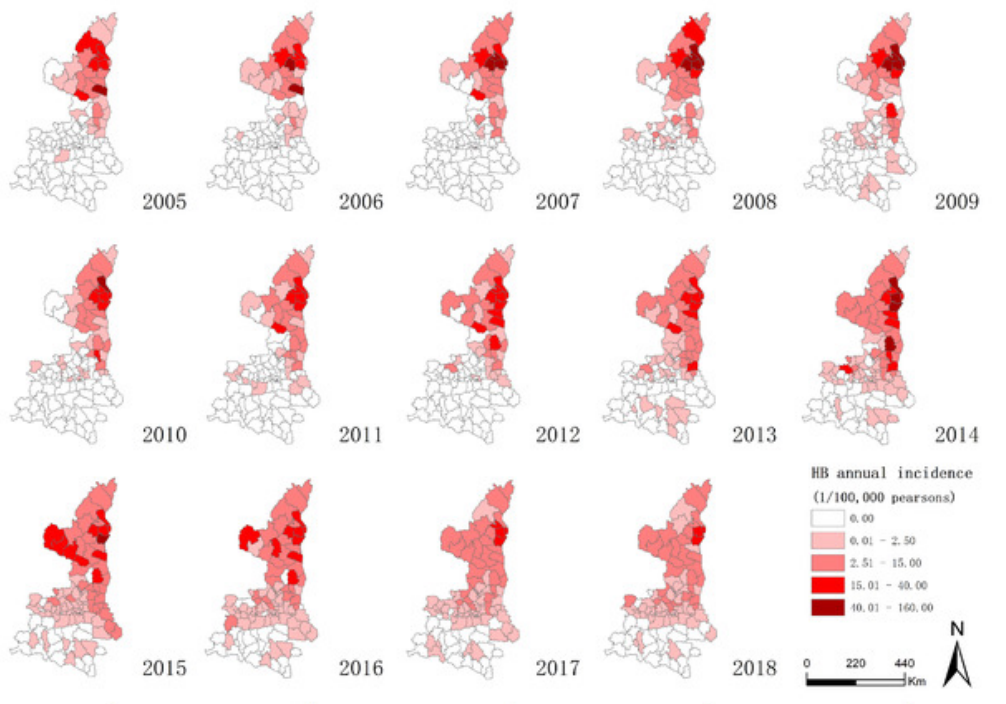

B
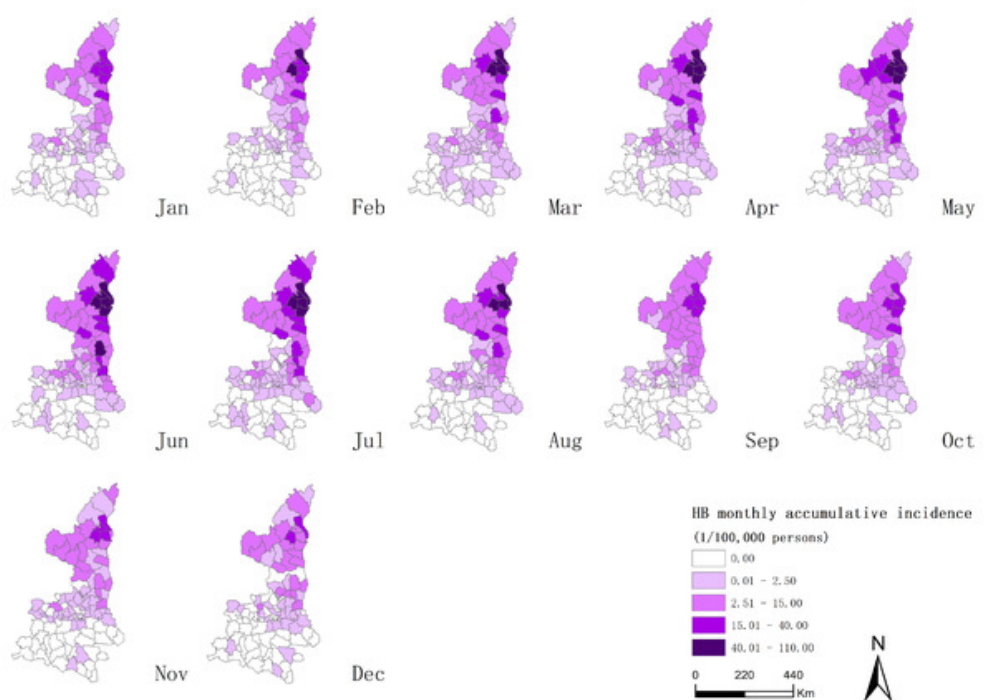

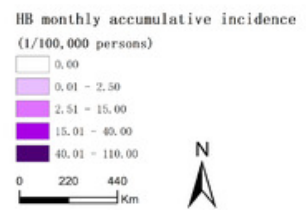

C

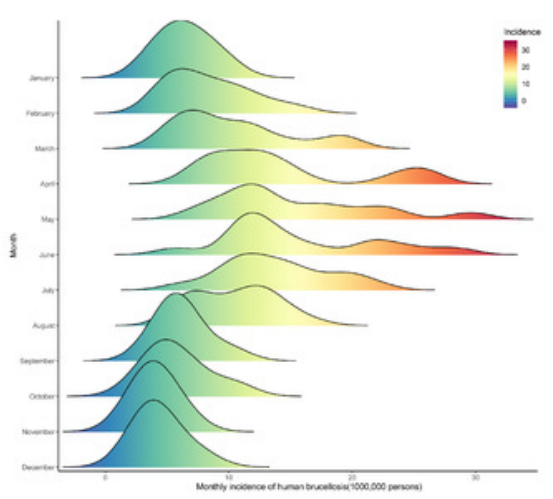

D

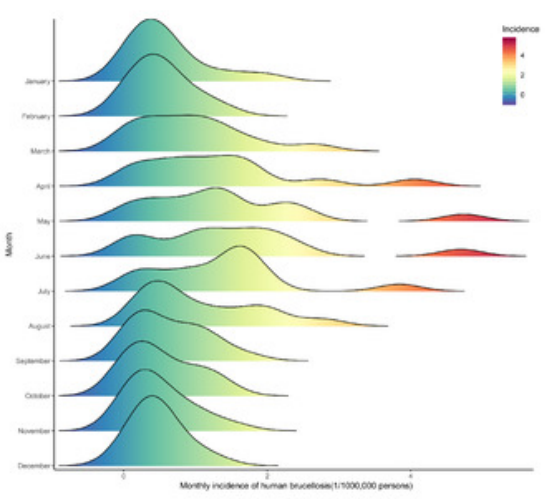


Figure 4

Figure 4. Observed and predicted numbers of human brucellosis cases (A), and the scatterplot (B) in Shaanbei upland plateau, 2005-2018.

A

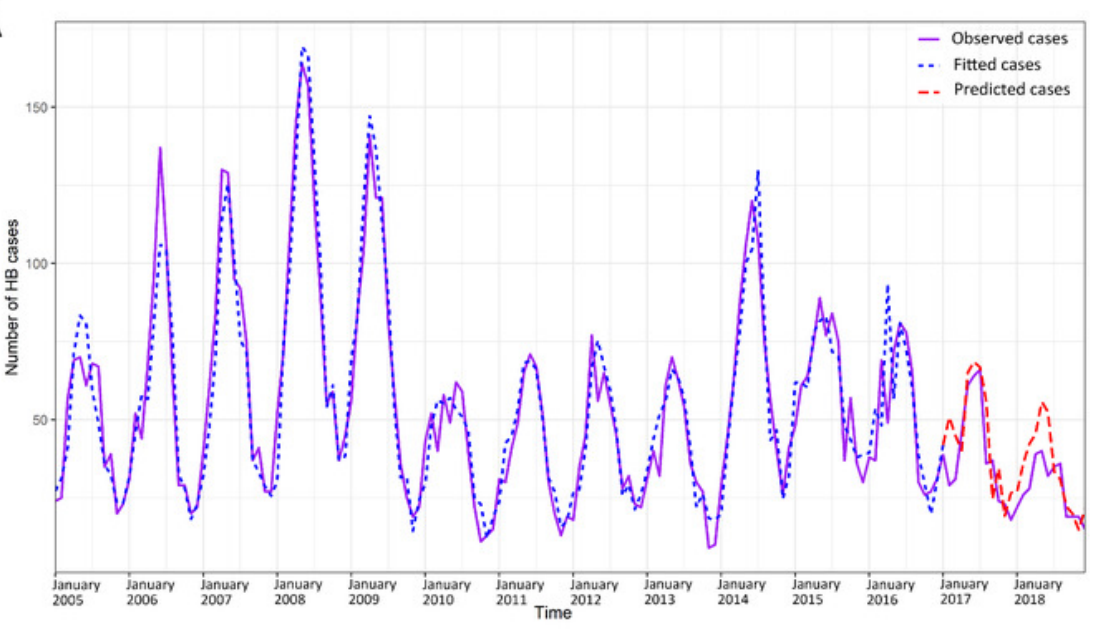

B

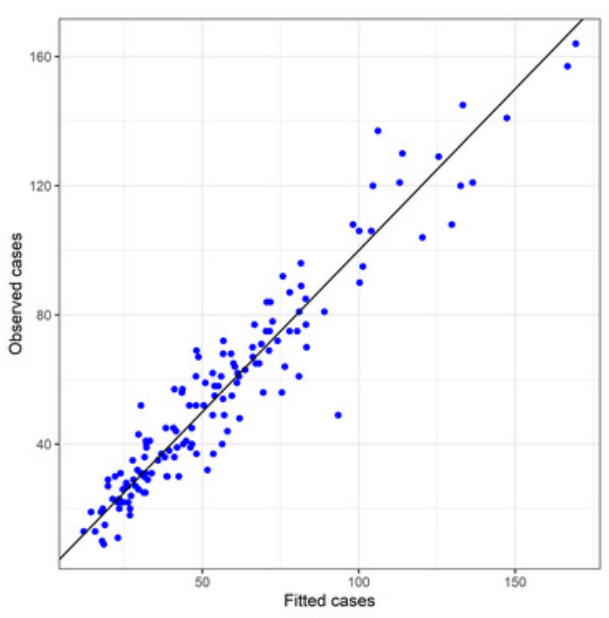


Figure 5

Figure 5. Observed and predicted numbers of human brucellosis cases (A), and the scatterplot (B) in Guanzhong basin, 2005-2018.
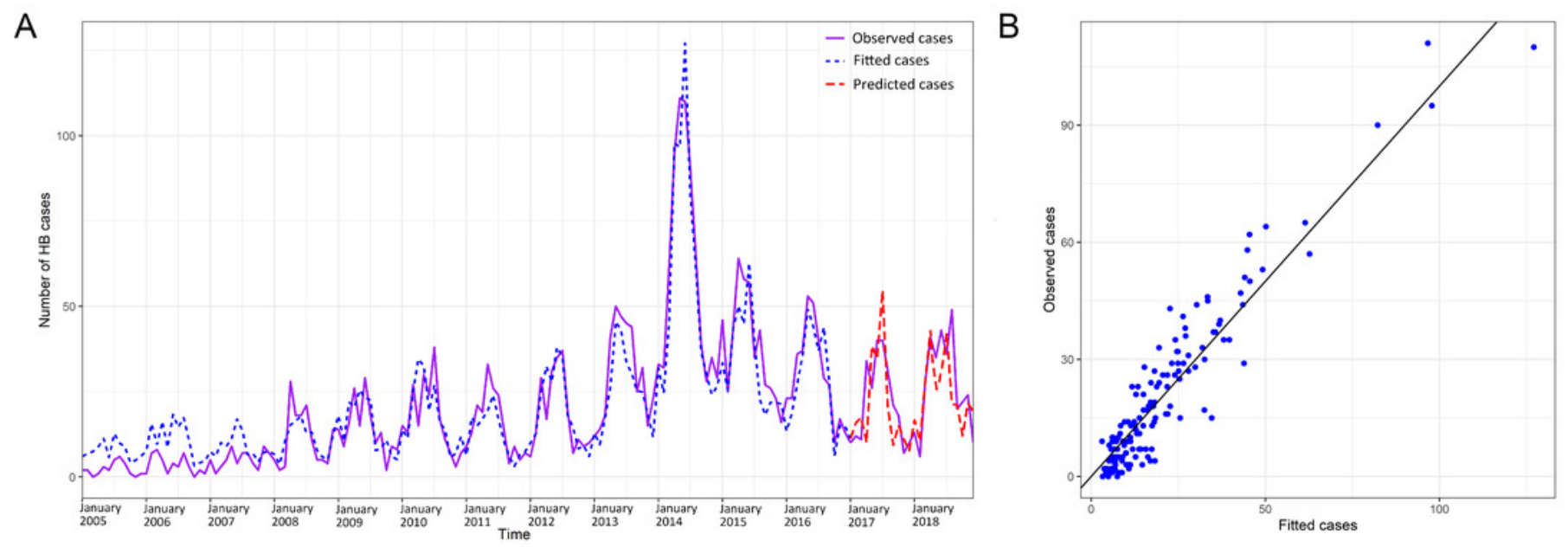
Figure 6

Figure 6. Relationship between number of human brucellosis cases and temperature $(A)$, relative humidity $(B)$, rainfall $(C)$, sunshine duration $(D)$, and evaporation $(E)$ in different regions.

Blue lines represent data for Shaanbei upland plateau, and red lines represent data for Guanzhong basin. 

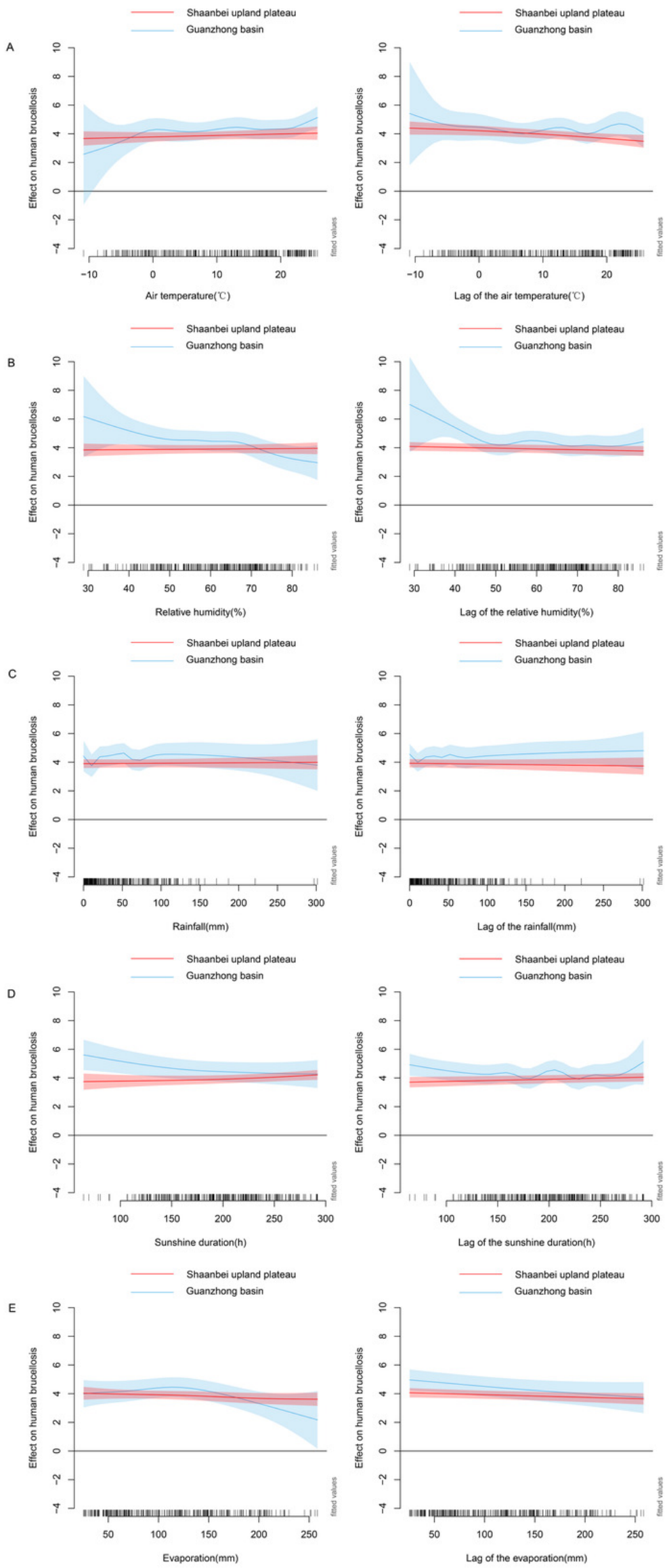

Peer] reviewing PDF | (2020:05:49509:1:1:NEW 15 Aug 2020) 